\section{Fatores de risco para o desmame entre usuárias de uma unidade básica de saúde de Belo Horizonte, Minas Gerais, Brasil, entre 1980 e 2004}

\author{
Risk factors for weaning among users of a primary \\ health care unit in Belo Horizonte, Minas Gerais \\ State, Brazil, from 1980 to 2004
}

Claudia Regina Lindgren Alves 1 Eugênio Marcos Andrade Goulart 1 Enrico Antônio Colosimo 2 Lúcia Maria Horta Figueiredo Goulart 1

\footnotetext{
${ }^{1}$ Faculdade de Medicina, Universidade Federal de Minas Gerais, Belo Horizonte, Brasil.

2 Instituto de Ciências Exatas, Universidade Federal de Minas Gerais, Belo Horizonte, Brasil.

Correspondência C. R. L. Alves Departamento de Pediatria Faculdade de Medicina, Universidade Federal de Minas Gerais. Av. Alfredo Balena 190, 4o andar, Belo Horizonte, $M G$ 30130-100, Brasil. lindgren@medicina.ufmg.br
}

\section{Abstract}

This study was a comparative analysis of factors affecting duration of breastfeeding among users of the São Marcos primary care clinic in Belo Horizonte, Minas Gerais State, Brazil, in 1980, 1986, 1992, 1998, and 2004. Five retrospective longitudinal studies (historical cohorts) were performed with the same questionnaire, and 790 mothers of children less than 24 months of age were interviewed. The statistical analysis was conducted year-by-year using the KaplanMeier method and Cox model. From 1980 to 2004, conditions significantly associated with risk of weaning were: primiparity; difficulty in postpartum breastfeeding; belief in ideal breastfeeding duration of less than six months; start of breastfeeding after discharge from the maternity hospital, non-recognition of the advantages of breastfeeding for the child; and unfavorable, unknown, or indifferent paternal opinion concerning breastfeeding. In four of the five studies, difficulty in breastfeeding (RR: 1.70-3.97) and belief in ideal breastfeeding duration of less than six months (RR: 1.67-3.27) were risk factors for weaning. Median duration of breastfeeding was five months in 1980 and 11 months in 2004.

Breast Feeding; Weaning; Health Promotion; Risk Factors
Introdução

O lema Saúde para Todos no Ano 2000, inaugurado na Conferência Internacional de Atenção Primária de Alma-Ata em 1978, foi a palavra de ordem da Organização Mundial da Saúde (OMS) para as últimas décadas do século XX. Países de todo o mundo perseguiram esse objetivo de acordo com suas realidades epidemiológicas, culturais e econômicas ${ }^{1}$. Uma das ações comuns a todos foi levantar a bandeira da promoção do aleitamento materno, como a estratégia capaz de reverter, por si só, as elevadas taxas de morbimortalidade infantil, especialmente nos países em desenvolvimento, e melhorar a qualidade de vida de mulheres e crianças 2 .

Há mais de 30 anos, a ciência vem dando sua contribuição para o resgate do aleitamento materno como prática indispensável à vida de mulheres e crianças. Passado todo esse tempo, não cabe mais discutir a propósito dos benefícios do aleitamento materno na prevenção de doenças, nos aspectos emocionais e financeiros. Muitas páginas poderiam ser preenchidas com evidências das qualidades nutricionais e imunológicas do leite materno, da importância da amamentação para o fortalecimento do vínculo afetivo mãe-filho e de outros incontáveis benefícios do aleitamento materno ${ }^{3}$.

Inúmeras maneiras de promover, estimular e apoiar o aleitamento materno vêm sendo experimentadas em todo o mundo. As principais 
estratégias de ação focalizam o acompanhamento pré-natal sistemático, a implantação do alojamento conjunto nas maternidades, o acompanhamento seqüencial da criança, a construção de creches e o respeito às leis de proteção à nutriz. Propaganda de massa, treinamento de profissionais de saúde, formação de grupos de gestantes e muitas outras estratégias procuram resgatar os benefícios do aleitamento materno para as mães e para as crianças 1,2. Os estudos registram, em vários países, o aumento progressivo de mulheres que optam por amamentar seus filhos e mostram que são raras as intervenções cujos resultados podem ser considerados inexpressivos 4,5. Promover o aleitamento materno tornou-se elemento fundamental das políticas públicas comprometidas com a qualidade de vida da população materno-infantil.

Numa primeira tentativa de cumprir no Brasil os compromissos assumidos em Alma-Ata, na então União Soviética, foi criado em 1981 o Programa Nacional de Incentivo ao Aleitamento Materno, com foco nas campanhas na mídia e na mobilização social e com o propósito de intervir nas causas de insucesso da amamentação. Foram criados comitês interinstitucionais cujas frentes de trabalho incluíam ações voltadas para capacitação dos estudantes/profissionais de saúde, estímulo às pesquisas e encontros científicos, reorganização dos serviços de saúde, revisão das leis trabalhistas e regulamentação da publicidade dos alimentos infantis industrializados 2,6.

Em 1984, incorporando os preceitos da OMS e do Fundo das Nações Unidas para a Infância (UNICEF), o Ministério da Saúde lançou o Programa de Atenção Integral à Saúde da Criança, definindo cinco ações básicas: (i) acompanhamento do crescimento e desenvolvimento, (ii) imunizações, (iii) controle de doenças diarréicas e terapia de reidratação oral, (iv) controle de doenças respiratórias agudas e (v) aleitamento materno e orientação alimentar para o desmame. Além de constituir-se em uma ação específica, a promoção do aleitamento materno foi fortemente valorizada nas demais ações desse programa e também no contemporâneo Programa de Atenção Integral à Saúde da Mulher. Desse modo, o incentivo ao aleitamento materno ganhou capilaridade e consistência nos serviços públicos e privados 6 .

Embora ainda deficiente, a Constituição $\mathrm{Fe}$ deral do Brasil de 1988 trouxe mudanças nas leis de proteção à gestante e à nutriz, que representaram um grande avanço na luta pela promoção do aleitamento materno. Outro passo importante na legislação nacional foi a aprovação em 1988 do primeiro texto da Norma Brasileira para Comercialização de Alimentos para Lactentes, que veio fortalecer o movimento pró-amamentação na medida em que criou limitações à propaganda e à produção de alimentos infantis industrializados 2 .

No início da década de 90, patrocinados pela OMS e pelo UNICEF, novos impulsos externos revitalizaram as ações pró-aleitamento materno no país. A Iniciativa Hospital Amigo da Criança foi implantada no Brasil a partir de 1992. Tornouse um importante mecanismo de promoção do aleitamento materno ao mobilizar funcionários de maternidades para a mudança de rotinas e atitudes, que contribuíam para os elevados índices de desmame precoce $6,7,8$.

Esse conjunto de ações resultou na retomada da prática do aleitamento materno no Brasil e no resgate de seu valor para a saúde de crianças e mulheres. A mediana nacional de duração do aleitamento materno quadriplicou em 25 anos 9 . Além disso, observou-se vertiginosa queda da mortalidade infantil no Brasil nos últimos 30 anos, especialmente a redução das mortes por desnutrição, doenças diarréicas e respiratórias, cuja relação com o aleitamento materno já foi exaustivamente estudada em todo o mundo $3,6,8$.

Muitas experiências regionais e locais de sucesso, como a participação de outros segmentos da sociedade, empresas públicas e privadas, entidades religiosas e meios de comunicação, têm criado uma atmosfera favorável à amamentação em todos os espaços da vida social. Lamentavelmente, a concretização do desejo de amamentar em nosso país, ainda esbarra em questões como a informalidade do trabalho feminino, a falta de equipamentos sociais de apoio à nutriz, a desestruturação do ambiente familiar e outras contradições de um país que vive sua transição rumo ao mundo desenvolvido.

Este estudo foi desenvolvido no Bairro São Marcos, uma comunidade tipicamente de periferia da região nordeste de Belo Horizonte, Estado de Minas Gerais, Brasil, que sofreu a influência dos fatores desestimulantes do aleitamento materno que prevaleceram nas décadas de 60 e 70 . A urbanização progressiva e rápida, a inserção cada vez maior da mulher no mercado de trabalho, a propaganda intensiva dos produtos industrializados e o apelo consumista influenciaram o pensamento daquela comunidade, assim como o fizeram com o restante do mundo ocidental. Essa comunidade recebeu também, a partir dos anos 80, a influência das propagandas de massa pró-aleitamento materno, das mudanças na legislação trabalhista e das políticas de saúde materno-infantil.

O impacto das muitas ações de promoção do aleitamento materno no modo de pensar e agir das mulheres do Bairro São Marcos em relação à 
alimentação de seus filhos, ao longo de 25 anos, foi o objeto do presente estudo. Procurou-se identificar as condições que interferiram na duração total da amamentação em cada ano e, com base nas lições aprendidas com esta história, construir estratégias de intervenção mais adequadas para a promoção do aleitamento materno.

\section{Metodologia}

Este estudo consta da análise comparativa de cinco estudos longitudinais retrospectivos (coortes históricas), realizados na área de abrangência do Centro de Saúde São Marcos nos anos de 1980, 1986, 1992, 1998 e 2004. A definição clássica de estudo de coorte considera que o pesquisador escolhe um determinado grupo de pessoas livres de doença, que diferem entre si quanto à exposição a uma causa potencial de doença (fator de risco) e as acompanha ao longo do tempo, observando a ocorrência do evento. No caso da coorte histórica, o pesquisador observa no presente a exposição ocorrida ao longo de um determinado tempo do passado e, por isso, é considerado um estudo longitudinal, porém retrospectivo ${ }^{10}$. No presente trabalho, em cada ano, foram incluídas mulheres que, a partir do nascimento de seus filhos, tiveram a possibilidade de iniciar o aleitamento materno, cuja duração foi influenciada por uma série de fatores. Caracteriza-se, portanto, como uma coorte histórica. Esse tipo de delineamento e de análise vem sendo utilizado por diversos autores da área, mostrando-se adequado aos objetivos propostos 10,11,12.

O instrumento para coleta de dados 13 utilizado em 1980 serviu de base para a elaboração dos demais e era composto por questões abertas e fechadas, abordando aspectos sócio-econômicos, demográficos e assistenciais, e os conceitos relacionados à prática de aleitamento materno. As questões abertas não foram analisadas para este artigo. As variáveis analisadas são apresentadas nas Tabelas 1,2 e 3 .

Tabela 1

Características sócio-econômicas e demográficas das mães entrevistadas, e medianas * da duração do aleitamento materno em cada categoria (análise univariada), por ano do estudo. Centro de Saúde São Marcos, Belo Horizonte, Minas Gerais, Brasil, 1980-2004.

\begin{tabular}{|c|c|c|c|c|c|c|c|c|c|c|c|c|c|c|c|c|c|c|c|c|}
\hline \multirow[t]{2}{*}{ Variáveis explicativas } & \multicolumn{3}{|c|}{$\begin{array}{c}1980 \\
(n=143)\end{array}$} & \multirow[b]{2}{*}{$p * *$} & \multicolumn{4}{|c|}{$\begin{array}{c}1986 \\
(n=140)\end{array}$} & \multicolumn{4}{|c|}{$\begin{array}{c}1992 \\
(n=149)\end{array}$} & \multicolumn{4}{|c|}{$\begin{array}{c}1998 \\
(n=141)\end{array}$} & \multicolumn{4}{|c|}{$\begin{array}{c}2004 \\
(n=217)\end{array}$} \\
\hline & $\mathbf{n}$ & $\%$ & $\begin{array}{l}\text { medi- } \\
\text { ana }\end{array}$ & & $\mathbf{n}$ & $\%$ & $\begin{array}{c}\text { medi- } \\
\text { ana }\end{array}$ & $p^{* *}$ & $\mathbf{n}$ & $\%$ & $\begin{array}{l}\text { medi- } \\
\text { ana }\end{array}$ & $p^{\star \star}$ & $\mathbf{n}$ & $\%$ & $\begin{array}{c}\text { medi- } \\
\text { ana }\end{array}$ & $p^{\star \star}$ & $\mathbf{n}$ & $\%$ & $\begin{array}{l}\text { medi- } \\
\text { ana }\end{array}$ & $p^{* *}$ \\
\hline \multicolumn{21}{|l|}{ Idade da mãe (anos) } \\
\hline$\leq 19$ & 10 & 7,0 & 2,0 & 0,29 & 6 & 4,3 & 6,0 & 0,44 & 14 & 9,4 & 13,0 & 0,66 & 28 & 19,9 & 5,9 & 0,68 & 20 & 9,2 & 9,0 & 0,11 \\
\hline$>19$ & 133 & 93,0 & 5,0 & & 134 & 95,7 & 7,0 & & 135 & 90,6 & 7,0 & & 113 & 80,1 & 8,0 & & 197 & 90,8 & 11,0 & \\
\hline \multicolumn{21}{|l|}{$\begin{array}{l}\text { Escolaridade materna } \\
\text { (anos de estudo) }\end{array}$} \\
\hline$\leq 8$ & 132 & 92,3 & 5,0 & 0,07 & 130 & 92,9 & 7,0 & 0,72 & 110 & 73,8 & 7,0 & 0,56 & 101 & 71,6 & 8,0 & 0,38 & 107 & 49,3 & 11,0 & 0,49 \\
\hline$>8$ & 11 & 7,7 & 4,3 & & 10 & 7,1 & 3,0 & & 39 & 26,2 & 7,0 & & 40 & 28,4 & 14,0 & & 110 & 50,7 & 11,0 & \\
\hline \multicolumn{21}{|l|}{$\begin{array}{l}\text { Renda familiar mensal } \\
\text { (salários mínimos) }\end{array}$} \\
\hline$\leq 2$ & 112 & 78,3 & 6,0 & 0,23 & 116 & 82,9 & 6,0 & 0,95 & 80 & 53,7 & 9,0 & 0,23 & 70 & 49,6 & 10,0 & 0,35 & 126 & 58,1 & 11,0 & 0,60 \\
\hline$>2$ & 31 & 21,7 & 4,3 & & 24 & 17,1 & 6,0 & & 69 & 46,3 & 6,0 & & 71 & 50,4 & 5,9 & & 91 & 41,9 & 12,0 & \\
\hline \multicolumn{21}{|l|}{$\begin{array}{l}\text { Vínculo com o pai } \\
\text { da criança }\end{array}$} \\
\hline $\operatorname{Sim}$ & 127 & 88,8 & 5,0 & 0,84 & 127 & 90,7 & 12,0 & 0,74 & 129 & 86,6 & 6,0 & 0,77 & 133 & 94,3 & 5,0 & 0,27 & 188 & 86,6 & 5,0 & 0,03 \\
\hline Não & 16 & 11,2 & 7,0 & & 13 & 9,3 & 6,0 & & 20 & 13,4 & 7,0 & & 8 & 5,7 & 8,0 & & 29 & 13,4 & 12,0 & \\
\hline \multicolumn{21}{|l|}{ Número de filhos } \\
\hline Primíparas & 40 & 28,0 & 3,0 & 0,05 & 41 & 29,3 & $4,0<$ & $<0,001$ & 61 & 40,9 & 6,0 & 0,47 & 58 & 41,1 & $5,0<$ & $<0,001$ & 96 & 44,2 & 10,0 & 0,50 \\
\hline Multíparas & 103 & 72,0 & 7,0 & & 99 & 70,7 & 8,0 & & 88 & 59,1 & 8,0 & & 83 & 58,9 & 10,0 & & 121 & 55,8 & 11,0 & \\
\hline \multicolumn{21}{|l|}{ Sexo da criança } \\
\hline Masculino & 65 & 45,5 & 7,0 & 0,82 & 70 & 50,0 & 8,0 & 0,84 & 74 & 49,7 & 8,0 & 0,47 & 66 & 46,8 & 6,0 & 0,35 & 120 & 55,3 & 9,0 & 0,17 \\
\hline Feminino & 78 & 54,5 & 5,0 & & 70 & 50,0 & 6,0 & & 75 & 50,3 & 6,0 & & 75 & 53,2 & 9,0 & & 97 & 44,7 & 15,0 & \\
\hline
\end{tabular}

* Em meses;

** Log-rank. Em negrito, aquelas que compuseram o modelo inicial da análise multivariada de cada ano. 
Tabela 2

Características da assistência à gravidez, ao parto e ao puerpério recebida pelas mães entrevistadas, e medianas * da duração do aleitamento materno por categoria (análise univariada), por ano do estudo. Centro de Saúde São Marcos, Belo Horizonte, Minas Gerais, Brasil, 1980-2004.

\begin{tabular}{|c|c|c|c|c|c|c|c|c|c|c|c|c|c|c|c|c|c|c|c|c|}
\hline \multirow[t]{2}{*}{ Variáveis explicativas } & \multicolumn{4}{|c|}{$\begin{array}{c}1980 \\
(n=143)\end{array}$} & \multicolumn{4}{|c|}{$\begin{array}{c}1986 \\
(n=140)\end{array}$} & \multicolumn{4}{|c|}{$\begin{array}{c}1992 \\
(n=149)\end{array}$} & \multicolumn{4}{|c|}{$\begin{array}{c}1998 \\
(n=141)\end{array}$} & \multicolumn{4}{|c|}{$\begin{array}{c}2004 \\
(n=217)\end{array}$} \\
\hline & $\mathbf{n}$ & $\%$ & $\begin{array}{c}\text { medi- } \\
\text { ana }\end{array}$ & $-p^{* *}$ & $\mathbf{n}$ & $\%$ & $\begin{array}{l}\text { medi- } \\
\text { ana }\end{array}$ & $p^{* *}$ & $\mathbf{n}$ & $\%$ & $\begin{array}{l}\text { medi- } \\
\text { ana }\end{array}$ & $p * *$ & $\mathbf{n}$ & $\%$ & $\begin{array}{c}\text { medi- } \\
\text { ana }\end{array}$ & $p * *$ & $\mathbf{n}$ & $\%$ & $\begin{array}{l}\text { medi- } \\
\text { ana }\end{array}$ & $p^{\star \star}$ \\
\hline \multicolumn{21}{|l|}{ Realização do } \\
\hline \multicolumn{21}{|l|}{ pré-natal } \\
\hline Não & 10 & 7,0 & 16,0 & 0,37 & 5 & 3,6 & 6,0 & 0,83 & 3 & 2,0 & 4,0 & 0,47 & 1 & 0,7 & $\star \star \star *$ & 0,73 & 0 & 0,0 & - & - \\
\hline Sim & 133 & 93,0 & 5,0 & & 135 & 96,4 & 7,0 & & 146 & 98,0 & 7,0 & & 140 & 99,3 & 8,0 & & 217 & 100,0 & & \\
\hline \multicolumn{21}{|l|}{$\begin{array}{l}\text { Orientação para ama- } \\
\text { mentar no pré-natal }\end{array}$} \\
\hline Não & 47 & 32,9 & 6,0 & 0,88 & 48 & 34,3 & 6,0 & 0,85 & 57 & 38,3 & 14,0 & 0,01 & 51 & 36,2 & 14,0 & 0,27 & 81 & 37,3 & 15,0 & 0,47 \\
\hline Sim & 96 & 67,1 & 5,0 & & 92 & 65,7 & 6,0 & & 92 & 61,7 & 6,0 & & 90 & 63,8 & 7,0 & & 136 & 62,7 & 11,0 & \\
\hline \multicolumn{21}{|l|}{$\begin{array}{l}\text { Orientação para ama- } \\
\text { mentar na maternidade }\end{array}$} \\
\hline Não & 75 & 52,5 & 4,0 & 0,05 & 35 & 25,0 & 7,0 & 0,89 & 62 & 41,6 & 12,0 & 0,03 & 34 & 24,1 & 5,0 & 0,09 & 26 & 11,9 & 13,0 & 0,69 \\
\hline Sim & 68 & 47,5 & 8,0 & & 105 & 75,0 & 6,0 & & 87 & 58,4 & 6,0 & & 107 & 75,9 & 7,0 & & 191 & 88,1 & 9,0 & \\
\hline \multicolumn{21}{|l|}{ Início do aleitamento } \\
\hline \multicolumn{21}{|l|}{ materno (exclusivo ou } \\
\hline \multicolumn{21}{|l|}{ não) na maternidade } \\
\hline Não & 69 & 48,3 & 2,0 & $<0,001$ & 16 & 11,4 & 2,0 & $<0,001$ & 70 & 46,9 & 7,0 & 0,89 & 21 & 14,9 & 4,0 & 0,16 & 11 & 5,1 & 6,0 & 0,83 \\
\hline Sim & 74 & 51,7 & 11,0 & & 124 & 88,6 & 8,0 & & 79 & 53,1 & 6,0 & & 120 & 85,1 & 8,0 & & 206 & 94,9 & 11,0 & \\
\hline \multicolumn{21}{|l|}{ Tempo de separação } \\
\hline \multicolumn{21}{|l|}{ pós-parto (horas) } \\
\hline$\geq 6$ & 127 & 88,8 & 5,0 & 0,62 & 94 & 67,1 & 6,0 & 0,52 & 94 & 63,1 & 8,0 & 0,58 & 73 & 51,7 & 6,0 & 0,02 & 49 & 22,6 & 16,0 & 0,21 \\
\hline$<6$ & 16 & 11,2 & 7,0 & & 46 & 32,9 & 7,0 & & 55 & 36,9 & 6,0 & & 68 & 48,3 & 12,0 & & 168 & 77,4 & 10,0 & \\
\hline $\begin{array}{l}\text { Local de permanência } \\
\text { do recém-nascido } \\
\text { após o parto }\end{array}$ & & & & & & & & & & & & & & & & & & & & \\
\hline $\begin{array}{l}\text { No berçário por } \\
\text { mais de } 1 \text { dia }\end{array}$ & 82 & 57,3 & 4,0 & & 85 & 60,7 & 8,0 & & 68 & 45,6 & 7,0 & & 41 & 29,1 & 7,0 & & 30 & 13,8 & 9,0 & \\
\hline $\begin{array}{l}\text { No berçário por } \\
1 \text { dia ou menos }\end{array}$ & 61 & 42,7 & 1,0 & 0,07 & 30 & 21,5 & 5,0 & 0,23 & 36 & 24,2 & 6,0 & 0,47 & 29 & 20,5 & 8,0 & 0,76 & 10 & 4,6 & 8,0 & 0,87 \\
\hline $\begin{array}{l}\text { Alojamento } \\
\text { conjunto }\end{array}$ & 0 & 0,0 & - & & 25 & 17,8 & 10,0 & & 45 & 30,2 & 12,0 & & 71 & 50,4 & 8,0 & & 177 & 81,6 & 11,0 & \\
\hline \multicolumn{21}{|l|}{$\begin{array}{l}\text { Dificuldade para ama- } \\
\text { mentar no pós-parto }\end{array}$} \\
\hline Sim & 50 & 35,0 & 2,0 & $<0,001$ & 44 & 31,4 & 3,0 & $<0,001$ & 64 & 42,9 & $4,0<$ & $<0,001$ & 63 & 44,7 & $4,0<$ & $<0,001$ & 86 & 39,6 & 7,0 & 0,04 \\
\hline Não & 93 & 65,0 & 12,0 & & 96 & 68,6 & 8,0 & & 85 & 57,1 & 12,0 & & 78 & 55,3 & 11,5 & & 131 & 60,4 & 12,0 & \\
\hline \multicolumn{21}{|l|}{ Peso de nascimento } \\
\hline$<2.500$ & - & - & - & - & - & - & - & - & - & - & - & - & 14 & 9,9 & 8,0 & 0,41 & 26 & 12,0 & 16,0 & 0,36 \\
\hline$\geq 2.500$ & - & - & - & - & - & - & - & - & - & - & - & - & 127 & 90,1 & 8,0 & & 191 & 88,0 & 9,0 & \\
\hline \multicolumn{21}{|l|}{ Tipo de parto \# } \\
\hline Cesárea & - & - & - & - & - & - & - & - & - & - & - & - & 55 & 39,0 & 5,9 & 0,37 & 70 & 32,3 & 9,0 & 0,54 \\
\hline Normal & - & - & - & - & - & - & - & - & - & - & - & - & 86 & 61,0 & 10,0 & & 147 & 67,7 & 9,0 & \\
\hline
\end{tabular}

* Em meses;

** Log-rank. Em negrito, aquelas que compuseram o modelo inicial da análise multivariada;

*** Não foi possível calcular a mediana, pois todas as crianças da categoria ainda estavam sendo amamentadas na data da entrevista (dados censurados);

\# Dados coletados apenas em 1998 e 2004. 
Características dos conceitos relacionados à prática da amamentação e medianas * da duração do aleitamento materno por categoria (análise univariada), por ano do estudo. Centro de Saúde São Marcos, Belo Horizonte, Minas Gerais, Brasil, 1980-2004.

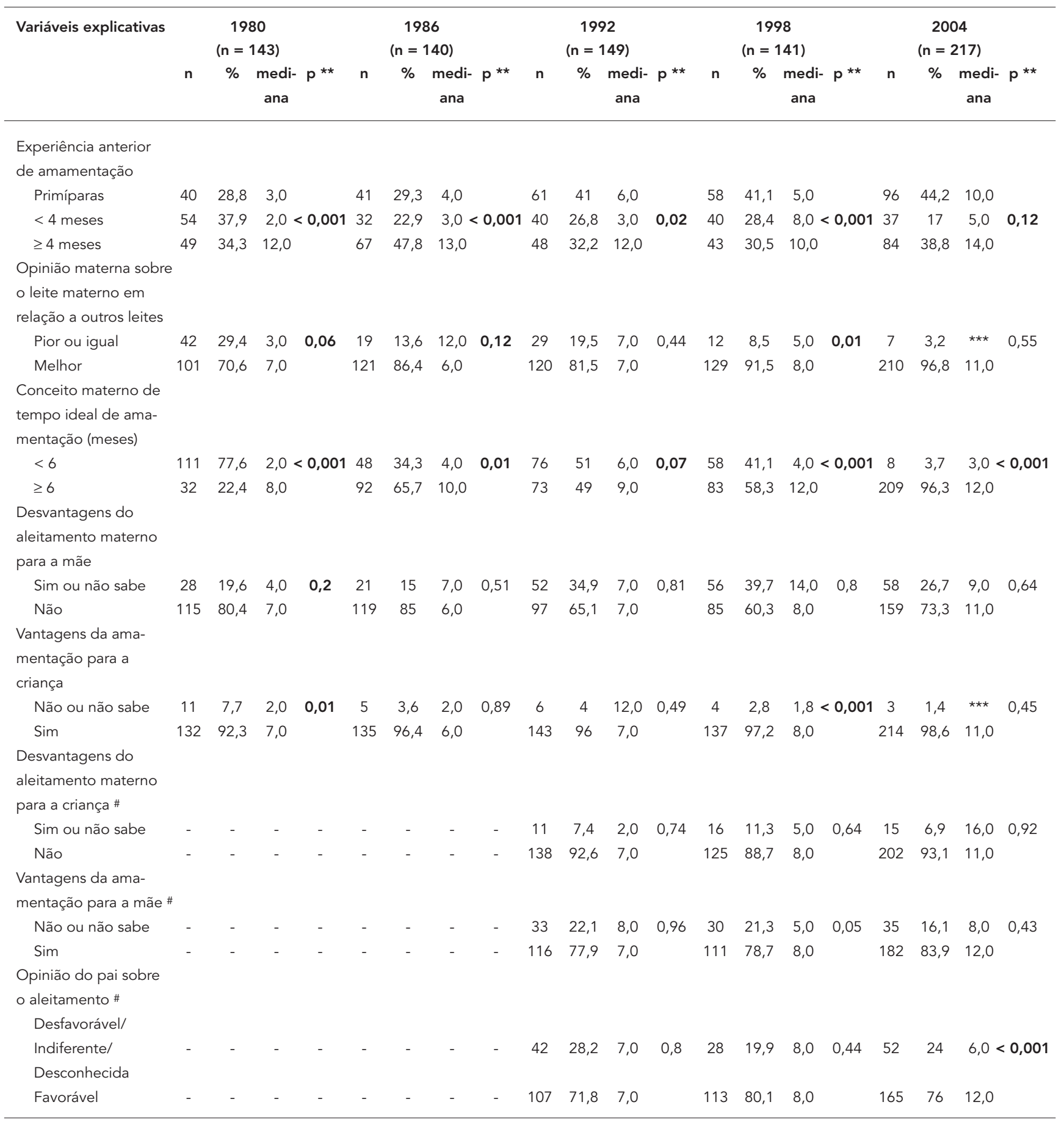

$\star$ Em meses;

** Log-rank. Em negrito, aquelas que compuseram o modelo inicial da análise multivariada;

*** Não foi possível calcular a mediana, pois todas as crianças da categoria ainda estavam sendo amamentadas na data da entrevista (dados censurados);

\# Dados coletados a partir de 1992. 
Foram incluídas no estudo usuárias do Centro de Saúde São Marcos, cujos filhos tinham idade inferior a 24 meses e que tiveram a possibilidade de iniciar o aleitamento materno. Cada mulher foi entrevistada apenas uma vez e as perguntas dirigidas ao filho mais novo. Em todos os anos foram excluídas as mães adotivas e aquelas com contra-indicação formal para amamentar.

As entrevistas foram realizadas no Centro de Saúde São Marcos, que foi construído em 1976 pela Secretaria Municipal de Saúde de Belo Horizonte (SMS-BH). A partir de 1979, tornou-se um centro de treinamento em atenção primária para estudantes da área da saúde da Universidade Federal de Minas Gerais (UFMG), em especial os de medicina. Atualmente, o Centro de Saúde São Marcos é responsável por aproximadamente 3.500 famílias.

Nos estudos de 1980 a 1998, as entrevistas foram realizadas por um professor do Departamento de Pediatria, Faculdade de Medicina/ UFMG e seus alunos. As mães foram convidadas a participar da pesquisa no momento em que compareciam ao Centro de Saúde São Marcos, por motivos variados, para utilização de seus serviços. Foram entrevistadas as primeiras 150 mulheres que preenchessem os critérios de inclusão. Nesses estudos, não foi possível o dimensionamento do tamanho da população a ser estudada, devido à ausência de um sistema de informação estruturado e ao modelo assistencial adotado na época. A escolha do tamanho da amostra foi baseada nas possibilidades operacionais dos pesquisadores. Seguiu-se a mesma distribuição das crianças por faixa etária definida no primeiro estudo.

Em 2004, as entrevistas foram feitas pelos agentes comunitários de saúde do Centro de Saúde São Marcos, que receberam treinamento específico para esta tarefa. Desde 2002, o modelo assistencial vigente na rede básica de saúde de Belo Horizonte é o do Programa Saúde da Família. Isso permitiu a delimitação da população adscrita ao Centro de Saúde São Marcos e possibilitou a identificação de todas as usuárias do serviço que preenchiam os critérios de inclusão no estudo. Nesse ano, foi realizado um estudopiloto em que cada agente comunitário de saúde realizou cinco entrevistas, que foram conferidas integralmente pelo telefone por estudantes de medicina. Em seguida, os agentes comunitários de saúde receberam novo treinamento reforçando os pontos em que surgiram dificuldades de preenchimento. Os agentes comunitários de saúde entrevistaram todas as 222 mães que preenchiam os critérios de inclusão na área de abrangência do Centro de Saúde São Marcos, com exceção de uma que recusou-se a participar e quatro mães que não foram localizadas, o que representou $2,3 \%$ de perdas. Cerca de $20 \%$ do total de entrevistas, em 2004, foram conferidos total ou parcialmente pelo telefone.

As análises foram realizadas ano a ano a partir de um banco de dados criado no programa Epi Info versão 6.04 (Centers for Disease Control and Prevention, Atlanta, Estados Unidos). Foram utilizados os mesmos pontos de corte e categorias em todos os estudos, cuja definição baseou-se nas características da população estudada e nas referências clássicas da literatura, conforme apresentado nas Tabelas 1, 2 e 3 . Apenas os questionários com todas as variáveis explicativas devidamente preenchidas foram incluídos no banco de dados, o que provocou algumas perdas. Em 1980, foram excluídos 5,3\% dos questionários com problemas de preenchimento que inviabilizavam as análises estatísticas. Esses valores foram $6,6 \%$ em 1986, $2 \%$ em 1992 e 7,8\% em 1998. Em 2004, foram aproveitadas todas as entrevistas realizadas.

Os dados de cada ano foram submetidos aos mesmos procedimentos estatísticos, cuja seqüência foi uma análise exploratória inicial, uma análise univariada pelo método de Kaplan-Meier e uma análise multivariada pelo modelo de regressão de Cox. Os resultados dos modelos finais da análise multivariada foram discutidos comparativamente à luz das modificações sócio-econômicas e demográficas observadas na população e da evolução das políticas públicas de saúde para a população materno-infantil implementadas ao longo dos 25 anos de observação.

As variáveis explicativas foram agrupadas em três categorias: sócio-econômicas e demográficas, assistência na gravidez, parto e puerpério e conceitos relacionados às práticas do aleitamento materno, conforme mostrado nas Tabelas 1, 2 e 3, respectivamente. Essa última categoria incluiu variáveis como a experiência anterior de amamentação da mãe, suas concepções sobre aleitamento materno e a opinião do pai sobre o tema. Foram feitas distribuições de freqüência dessas variáveis para construção do perfil das mães em cada ano.

Foram estudados os fatores relacionados à duração do uso de leite materno, associado ou não a qualquer outro tipo de alimento, padrão definido como aleitamento materno pela OMS, conforme citado por Giugliani 14. Considerou-se desmame total quando a criança deixou de receber definitivamente o leite materno. A variável resposta (evento final) empregada nas análises univariada e multivariada foi o desmame total.

$\mathrm{Na}$ análise univariada foram construídas curvas de sobrevida da duração do aleitamento materno para todas as variáveis explicativas 
das três categorias em cada um dos estudos, e a comparação entre as mães que ainda estavam amamentando e que já haviam desmamado foi realizada pelo teste de log-rank, calculado a partir do estimador não-paramétrico de KaplanMeier 15. A mediana da duração do aleitamento materno em cada estudo também foi estimada utilizando-se esse método.

O modelo de regressão de riscos proporcionais (Cox) foi utilizado para determinar o efeito independente das variáveis selecionadas sobre o desmame. Os modelos iniciais da análise multivariada, em cada estudo, incluíram variáveis que apresentaram $\mathrm{p} \leq 0,20$ (log-rank) na análise univariada nas três categorias. A estratégia de construção dos modelos foi a backward elimination 16. O processo de exclusão das variáveis foi encerrado quando o valor de $\mathrm{p}$ de todas as variáveis de um mesmo modelo era menor que 0,05 . Foi testada a ocorrência de interações entre as co-variáveis de cada modelo final pelo mesmo método. O pressuposto de proporcionalidade dos riscos do modelo de Cox foi verificado pela observação dos gráficos log minus log das variáveis com significância estatística em função do tempo, derivados das curvas de sobrevida (Kaplan-Meier). As variáveis cujas curvas de seus estratos não se cruzavam foram assumidas como independentes em relação ao tempo e mantidas no modelo final de Cox 17. Considerou-se o intervalo de confiança de $95 \%$ no cálculo do risco relativo (RR).

Este projeto foi aprovado pelo Comitê de Ética em Pesquisa do Hospital das Clínicas da UFMG e pela Câmara do Departamento de Pediatria da Faculdade de Medicina da UFMG.

\section{Resultados}

As entrevistadas tinham entre 26 e 28 anos, em média. As mães adolescentes compreendiam cerca de $20 \%$ das entrevistadas em 1998, caindo para $9 \%$ em 2004. Apesar da melhoria da escolaridade das mães, em 2004 apenas metade das entrevistadas havia concluído o ensino fundamental e quase $60 \%$ das famílias tinham uma renda mensal igual ou inferior a dois salários mínimos. Ao longo dos anos, observou-se aumento do número de primíparas. Cerca de $90 \%$ das entrevistadas relataram ter algum tipo de vínculo com o pai de seus filhos em todos os anos. A Tabela 1 mostra as características sócio-econômicas e demográficas das entrevistadas de 1980 a 2004.

As características da assistência recebida pelas mães na gravidez, parto e puerpério, segundo o ano do estudo, são apresentadas na Tabela 2. Houve aumento progressivo do percentual de mães que realizaram o pré-natal, chegando a $100 \%$ em 2004. No entanto, o percentual de mães que foram orientadas a amamentar no pré-natal não aumentou significativamente entre 1980 e 2004 e correspondeu a menos de $70 \%$. O percentual de mães que receberam orientação para amamentar na maternidade passou de $47,5 \%$ em 1980 para $88,1 \%$ em 2004 . No primeiro estudo, nenhuma mãe ficou em alojamento conjunto com seus filhos e mais da metade dos recém-nascidos ficaram por mais de um dia no berçário. Em 2004, mais de $80 \%$ das entrevistadas ficaram em alojamento conjunto após o parto. Entre 1980 e 2004, houve aumento importante do percentual de recém-nascidos que iniciaram o aleitamento materno (exclusivo ou não) ainda na maternidade e diminuição do percentual de crianças que ficaram separadas de suas mães por seis horas ou mais após o parto. O percentual de mães que apresentaram dificuldades para amamentar nos primeiros dias após o parto variou de $31,4 \%$ a $44,7 \%$ entre os anos de observação.

Na Tabela 3, são apresentadas as características dos conceitos relacionados às práticas de aleitamento materno, em cada ano estudado. Entre 1980 e 2004, houve aumento do percentual de mães que consideram o leite materno melhor que os outros tipos de leite, que acham ideal amamentar por seis meses ou mais, que conseguiram amamentar seus filhos anteriores por pelo menos quatro meses e que identificam vantagens do leite materno para a criança. As variáveis "desvantagens do aleitamento materno para a criança", "vantagens da amamentação para a mãe" e "opinião do pai sobre o aleitamento materno" só foram coletadas nos anos de 1992, 1998 e 2004 e não foram observadas diferenças importantes entre os estudos.

A mediana da duração do aleitamento materno passou de 5 para 11 meses entre o primeiro e o último estudo. A Figura 1 mostra as curvas de sobrevida para a duração do aleitamento materno, segundo o ano do estudo ( $\mathrm{p}<0,001, \log$-rank). Entre 1980 e 2004, observou-se aumento progressivo da prevalência do aleitamento materno em todas as idades. Mais de $20 \%$ das crianças menores que um mês já haviam desmamado em 1980 e, em 2004, menos de 5\% das crianças não recebiam mais leite materno nesta idade.

Nas Tabelas 1, 2 e 3 são apresentadas as medianas da duração do aleitamento materno para as variáveis explicativas estudadas e o valor de $p$ (teste $\log$-rank), calculado pelo método de Kaplan-Meier em cada ano. As variáveis que apresentaram $\mathrm{p} \leq 0,20$ estão em negrito e foram incluídas no modelo inicial da análise multivariada de cada estudo. Observou-se tendência de aumento da mediana de duração do aleitamento materno, 
Curvas de sobrevida para a duração total do aleitamento materno por ano do estudo. Centro de Saúde São Marcos, Belo Horizonte, Minas Gerais, Brasil, 1980-2004.

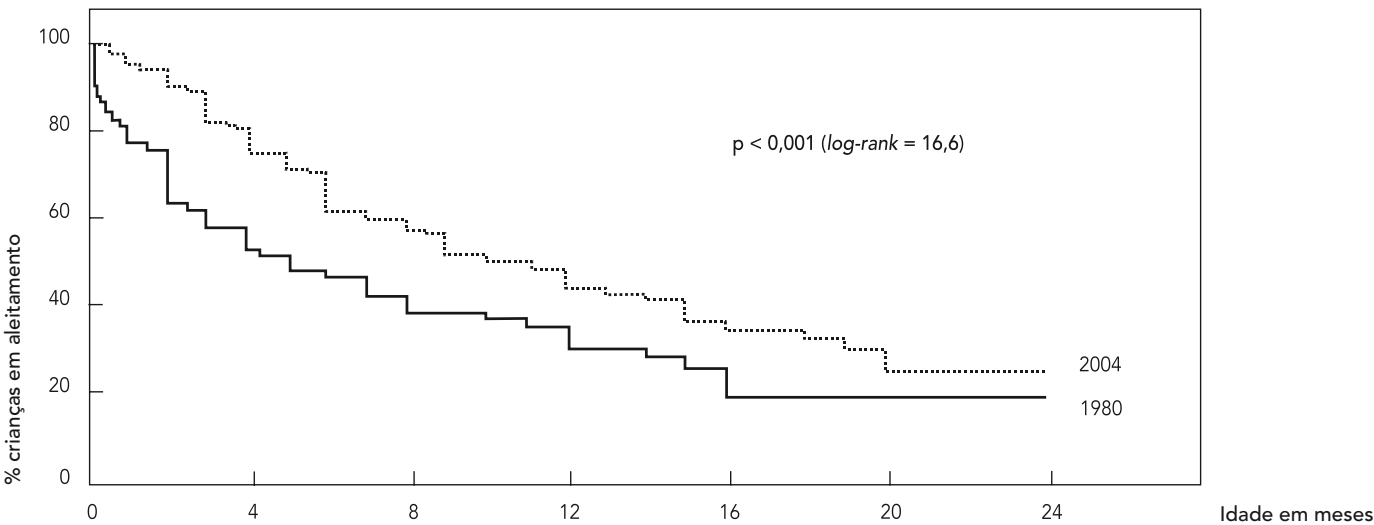

entre 1980 e 2004 , em praticamente todas as variáveis analisadas.

A Tabela 4 apresenta os modelos finais da análise multivariada pelo método de Cox, por ano do estudo, com os riscos relativos (RR), intervalos de confiança e valores de p, para cada uma das variáveis com significância estatística nos respectivos modelos finais. O conjunto de variáveis independentemente associadas à duração do aleitamento materno diferiu de ano para ano.

As condições associadas ao risco de menor duração do aleitamento materno, entre 1980 e 2004, foram: primiparidade, dificuldade para amamentar nos primeiros dias após o parto, tempo ideal de aleitamento materno menor que seis meses, início do aleitamento materno após a alta da maternidade, não reconhecimento das vantagens da amamentação para a criança e opinião paterna desfavorável, indiferente ou desconhecida sobre o aleitamento materno. $\mathrm{O}$ fato da mãe ter tido dificuldade para amamentar (RR: $1,70-3,97)$ e de considerar ideal amamentar por menos de seis meses (RR: 1,67-3,27) representaram risco de menor duração do aleitamento em quatro dos cinco anos estudados (Tabela 4).

A interação entre as co-variáveis do modelo final de cada estudo foi testada e apenas em 1998 houve associação significativa entre as variáveis "tempo ideal de amamentação" e "dificuldade para amamentar no pós-parto imediato" (RR: 5,44; IC: 1,87-15,8). Nesse ano, o efeito da interação potencializou o risco de desmame para as mães que acreditavam que o tempo ideal de amamentação era inferior a seis meses e também tiveram dificuldade para amamentar e vice-versa. Isso significa que, quando a mãe acreditava que o tempo ideal de amamentação era inferior a seis meses, o risco de desmame para aquelas com dificuldade de amamentar foi 17,5 vezes o risco daquelas sem dificuldade para amamentar.

\section{Discussão}

Vários desafios metodológicos se apresentaram para a realização deste trabalho. Idealmente, a composição das amostras deve levar em consideração seu tamanho e representatividade 10,18. Foram feitas, a posteriori, estimativas do tamanho e das características da população à qual as entrevistadas pertenciam com base nos censos demográficos, em informações da SMS-BH e em levantamentos da cobertura vacinal e da triagem neonatal do Centro de Saúde São Marcos no período de 1980 a 1998. As evidências sugerem que a amostra estudada correspondia a $25 \%$ das crianças menores de 2 anos em 1980, 20\% em 1986, $23 \%$ em 1992 e cerca de $40 \%$ em 1998 e que várias características sócio-econômicas e demográficas da amostra eram semelhantes aos dados encontrados para Belo Horizonte em cada período, embora nenhum processo de randomização tenha sido adotado. O interesse inicial em 1980 era analisar a eficácia do programa de incentivo ao aleitamento materno desenvolvido pelo Cen- 
Modelos finais das análises multivariadas dos fatores de risco para o desmame total. Centro de Saúde São Marcos, Belo Horizonte, Minas Gerais, Brasil, 1980-2004.

\begin{tabular}{|c|c|c|c|c|}
\hline Ano & Co-variáveis com significância estatística & Valor de $p$ & RR & IC95\% \\
\hline \multirow[t]{5}{*}{1980} & Primíparas & 0,04 & 1,65 & $1,02-2,68$ \\
\hline & Mãe com dificuldade para amamentar no pós-parto imediato & 0,00 & 3,97 & $2,45-6,35$ \\
\hline & Recém-nascido não iniciou aleitamento materno na maternidade & 0,00 & 2,03 & $1,28-3,23$ \\
\hline & Tempo ideal de amamentação menor que 6 meses & 0,00 & 2,14 & $1,38-3,50$ \\
\hline & Ausência de vantagens da amamentação para a criança & 0,00 & 2,92 & $1,42-6,03$ \\
\hline \multirow[t]{3}{*}{1986} & Recém-nascido não iniciou aleitamento materno na maternidade & 0,00 & 3,92 & $2,08-7,38$ \\
\hline & Mãe com dificuldade para amamentar no pós-parto imediato & 0,03 & 1,70 & $1,04-2,79$ \\
\hline & Tempo ideal de amamentação menor que 6 meses & 0,04 & 1,67 & $1,02-2,74$ \\
\hline 1992 & Mãe com dificuldade para amamentar no pós-parto imediato & 0,00 & 2,36 & $1,46-3,83$ \\
\hline \multirow[t]{5}{*}{1998} & Ausência de vantagens da amamentação para a criança & 0,00 & 6,93 & $2,37-20,26$ \\
\hline & Mãe com dificuldade para amamentar no pós-parto imediato & 0,00 & 3,22 & $1,87-5,54$ \\
\hline & Tempo ideal de amamentação menor que 6 meses & 0,00 & 3,01 & $1,76-5,16$ \\
\hline & Interação entre dificuldade para amamentar nos primeiros dias após o parto e tempo & 0,00 & 5,44 & $1,87-15,84$ \\
\hline & ideal de amamentação * & & & \\
\hline \multirow[t]{2}{*}{2004} & Tempo ideal de amamentação menor que 6 meses & 0,01 & 3,27 & $1,31-8,15$ \\
\hline & $\begin{array}{l}\text { Pai da criança com opinião desfavorável ou indiferente sobre amamentação ou pai sem vínculo } \\
\text { com a mãe da criança }\end{array}$ & 0,01 & 1,86 & $1,19-2,90$ \\
\hline
\end{tabular}

* Ver descrição da interpretação nos Resultados.

tro de Saúde São Marcos 13, de modo que apenas mulheres que recebiam algum tipo de assistência nessa unidade de saúde poderiam ser incluídas. Nesse caso, uma distribuição aleatória de base territorial talvez não contemplasse este aspecto. Em todos os anos, atentou-se para que a idade dos filhos das entrevistadas obedecesse à mesma distribuição, o que evitou um possível viés de seleção.

O estudo procurou atender às regras básicas de elaboração e aplicação de questionários e/ou entrevistas, conforme sugerido por Barros \& Victora 18 para a realização de diagnósticos comunitários. O questionário foi elaborado e testado, procurando balancear questões abertas e fechadas, na tentativa de captar os aspectos objetivos e subjetivos relacionados à prática das mães. A montagem do banco de dados foi feita a partir dos originais das entrevistas desde 1980 até 2004, sendo os autores responsáveis por codificar as variáveis estudadas, conferir os dados digitados, verificar contradições e corrigir os possíveis erros, dando origem a um banco de dados consistente e uniforme.

As mudanças observadas nas características sócio-econômicas e demográficas das entrevistadas refletem o processo de urbanização por que passou Belo Horizonte e o país nas últimas décadas. Apesar das diferenças observadas en- tre os grupos de mulheres em cada ano, podese afirmar que estas não foram suficientemente grandes para provocar uma mudança de estrato social. Trata-se, ainda, de uma população pertencente aos estratos menos favorecidos da sociedade e, talvez, por isto, os resultados aqui apresentados não possam ser extrapolados para o conjunto da sociedade. Considerar esses aspectos é fundamental para a compreensão do arcabouço cultural que define e sustenta a decisão das mães de como alimentar seus filhos.

Embora muitos autores venham tentando estabelecer quais características sócio-econômicas e demográficas se comportam como fatores de risco para o desmame precoce, parece não haver consenso na literatura 19. Há evidências da influência da maternidade precoce, da baixa renda, da baixa escolaridade paterna e materna, do tipo de ocupação dos pais e de outros indicadores de uma condição de vida desfavorável sobre a duração do aleitamento materno 11,19,20,21,22. No entanto, neste estudo, nenhuma variável dessa categoria esteve associada ao risco de desmame precoce, com exceção do número de filhos das mulheres. Pode-se especular que os grupos de mulheres fossem muito homogêneos quanto a esses aspectos, não permitindo a detecção de diferenças estatisticamente significativas com o desenho metodológico adotado. No presente 
estudo, as primíparas de 1980 tiveram um risco 1,65 vez maior de desmamar precocemente do que as multíparas. É possível que essas primíparas tenham tido menor oportunidade de acesso às informações veiculadas na mídia e às ações de promoção do aleitamento materno do que as mães com mais filhos, que provavelmente já utilizavam o Centro de Saúde São Marcos há mais tempo.

Em 1980 e 1986, as mães que não iniciaram o aleitamento na maternidade apresentaram um risco 2 a 4 vezes maior de desmame do que quando saíam do hospital amamentando. Nos outros anos, esse resultado não se repetiu. As práticas adotadas nas maternidades em Belo Horizonte até a década de 90, como tempo prolongado de separação mãe-filho após o parto, a falta de alojamento conjunto e o uso sistemático de fórmulas infantis nos berçários, podem estar envolvidas com esses resultados. Observou-se aumento progressivo das condições favorecedoras do início precoce do aleitamento materno, principalmente a partir de 1992. Em 2004, quase a totalidade das crianças iniciou a amamentação antes da alta hospitalar.

Em Belo Horizonte, além das ações propostas pelos programas nacionais de saúde maternoinfantil, a partir de 1994 foi implantado um projeto de reestruturação da assistência à mulher e à criança, inspirado na Iniciativa Hospital Amigo da Criança, que interferiu substancialmente nas práticas das maternidades da rede pública do município 22. Desde 1998, as gestantes de baixo risco do Centro de Saúde São Marcos realizam seus partos preferencialmente na Maternidade Sophia Feldman, primeira a receber o título de Hospital Amigo da Criança em Belo Horizonte. As de alto risco gestacional são encaminhadas ao Hospital das Clínicas da UFMG, que historicamente se comprometeu com a adoção de práticas estimuladoras do aleitamento materno. Esses fatos parecem estar relacionados com as modificações observadas, entre 1998 e 2004, em quase todas as variáveis relacionadas à assistência à gravidez, ao parto e ao puerpério. No presente estudo, com exceção do início da amamentação na maternidade, outras variáveis dessa categoria não se mostraram associadas ao desmame precoce, diferentemente do que é relatado na literatura $14,19,20,23$.

$\mathrm{O}$ percentual de mães que informaram ter tido dificuldades para amamentar nos primeiros dias após o parto foi elevado em todos os anos. Neste estudo, ter tido dificuldade para amamentar representou risco de menor duração do aleitamento materno de 1980 a 1998, como também referido por outros autores 24,25. A dificuldade para amamentar no pós-parto representa o resulta- do de um conjunto de deficiências na orientação da mãe para aspectos práticos da amamentação, apontando falhas desde o preparo das mamas na gravidez até a intervenção precoce no pósparto 14. É, portanto, um bom indicador da qualidade da abordagem recebida pelas mães em relação ao aleitamento materno. A expansão da cobertura de pré-natal não reflete a melhoria da assistência recebida pelas gestantes. Não se observou aumento da proporção de mulheres que foram orientadas a amamentar durante o prénatal. Por outro lado, o percentual de mulheres que receberam orientação na maternidade sobre o aleitamento materno aumentou consideravelmente ao longo dos anos, chegando a quase $90 \%$ em 2004. Ainda hoje, a articulação entre as ações de promoção do aleitamento materno durante o pré-natal e o apoio à lactação no puerpério representam um grande desafio para as equipes de saúde.

A associação entre variáveis relacionadas aos conceitos das mães sobre o aleitamento materno e o desmame foi expressiva em todos os estudos. Em 1980 e 1998, o risco de interromper precocemente a amamentação para as mães que não identificavam vantagens do aleitamento materno para a criança foi maior do que para aquelas que reconheciam estas vantagens. Esse resultado pode ser entendido como uma das medidas do nível de informação da mãe sobre o aleitamento materno, cuja relação positiva com a duração da amamentação foi também demonstrada por outros autores 26. Em 2004, o risco de desmame foi maior quando o pai da criança tinha opinião desfavorável, indiferente ou desconhecida sobre amamentação do que quando ele era claramente favorável ao aleitamento materno. A atitude favorável e o apoio do pai da criança também favoreceram uma duração maior da amamentação nos estudos de Silveira \& Lamounier $11 \mathrm{e}$ Faleiros et al. 19.

O fato da mãe considerar ideal amamentar por menos de 6 meses esteve associado ao desmame precoce em 1980, 1986, 1998 e 2004. Diversos fatores podem atuar na formação do conceito de tempo ideal de amamentação entre as usuárias do Centro de Saúde São Marcos. Muito investimento tem sido feito em torno da importância do aleitamento materno exclusivo até o sexto mês de vida e, talvez, pouca atenção esteja sendo dada à idéia de que o aleitamento materno deva ser mantido até os dois anos de idade ou mais. Além disso, os obstáculos concretos à amamentação, como a necessidade de voltar ao trabalho e a falta de creches, podem criar uma expectativa de amamentar por menos tempo nas mulheres que se encontram nestas situações. É possível também que a cultura do desmame pre- 
coce ainda seja a dominante, sendo necessário repensar as formas de introduzir os conceitos fundamentais sobre aleitamento. Outros autores também demonstram a importância da intenção das mães de como e por quanto tempo amamentar na duração do aleitamento materno 19,27. Conceitos como esses são a base da construção da decisão da mãe de como alimentar seus filhos, cujo processo começa antes da concepção, torna-se muito intenso durante a gravidez e terá seu desfecho após o nascimento do bebê 28,29.

Em 1998, foi verificada associação significativa entre as variáveis "conceito de tempo ideal de amamentação" e "dificuldade para amamentar nos primeiros dias após o parto". Outros autores também demonstraram associação entre essas variáveis e a duração do aleitamento materno, embora não tenham sido encontradas pesquisas em que a interação entre elas fosse significativa 19,24,25,27. Essa interação reflete bem situações cotidianas da prática clínica e demonstra a importância de uma abordagem holística da amamentação, em que informação, orientação e apoio devem ser oferecidos de maneira harmônica, contínua e permanente, para que a mulher sinta-se segura de suas decisões e possa superar os obstáculos que surgirem durante o aleitamento materno 28,29 .

Entre 1980 e 2004, a mediana da duração total do aleitamento materno entre as usuárias do Centro de Saúde São Marcos passou de 5 para 11 meses. Embora a duração alcançada em 2004 seja menos da metade do proposto pela OMS, esses valores revelam avanços. Em 1975, a mediana da duração do aleitamento materno no Brasil era de 2,5 meses, passando para 5,5 meses em 1989, como resultado das campanhas de promoção da década de 80 30. Outros estudos encontraram valores igualmente baixos para a duração da amamentação em diversas partes nesse período 4,31. Na última pesquisa de caráter nacional realizada em 1999 nas capitais brasilei- ras e Distrito Federal, a mediana da duração foi de cerca de dez meses para o Brasil, oito meses para a Região Sudeste e sete meses para Belo Horizonte ${ }^{9}$. Estudos regionais também mostraram tendência de elevação da duração e da prevalência do aleitamento materno 21,23,31,32. As medianas de duração do aleitamento materno no Centro de Saúde São Marcos foram maiores do que outros estudos em Minas Gerais e capitais com perfil cultural semelhante 11,21,23,24,31. Possivelmente, além do impacto das ações de promoção desencadeadas no país e em Belo Horizonte, as intervenções locais desenvolvidas pelos profissionais do Centro de Saúde São Marcos, entre eles professores e acadêmicos da UFMG, possam ter representado um estímulo a mais para a prática do aleitamento materno entre suas usuárias. Há que se considerar ainda possíveis interferências metodológicas nos resultados encontrados.

Superar os obstáculos para o aleitamento materno depende da capacidade dos profissionais/serviços de saúde adequarem suas práticas ao contexto sócio-demográfico e epidemiológico atual, otimizando as possibilidades que o modelo assistencial vigente oferece de apoiar efetivamente as mulheres em sua decisão de amamentar seus filhos. É necessário repensar a promoção do aleitamento materno quanto à abordagem das gestantes, despertando nelas o desejo de amamentar por pelo menos dois anos. As intervenções devem ainda propiciar às mães oportunidades de adquirir habilidades práticas para o aleitamento, minimizando as dificuldades iniciais.

Para uma abordagem mais profunda do assunto serão necessários estudos que permitam explorar o processo de tomada de decisão das mães sobre como alimentar seus filhos, bem como suas reais necessidades de apoio/suporte por parte dos profissionais de saúde, dos familiares e da sociedade em geral. 


\section{Resumo}

O objetivo foi analisar comparativamente os fatores que interferiram na duração do aleitamento materno entre usuárias do Centro de Saúde São Marcos, Belo Horizonte, Estado de Minas Gerais, Brasil, em 1980, 1986, 1992, 1998 e 2004. Foram realizados cinco estudos longitudinais retrospectivos (coortes históricas), utilizando o mesmo questionário. Foram entrevistadas, no total, 790 mães de menores de 24 meses. A análise estatística foi feita, ano a ano, pelo método de Kaplan-Meier e modelo de Cox. Entre 1980 e 2004, as condições significativamente associadas ao risco de desmame foram: primiparidade, dificuldade para amamentar após o parto, conceito de tempo ideal de aleitamento materno menor que seis meses, início do aleitamento materno após a alta da maternidade, não reconhecimento das vantagens da amamentação para a criança e opinião paterna desfavorável, indiferente ou desconhecida sobre o aleitamento materno. Em quatro dos cinco estudos, ter tido dificuldade para amamentar (RR: 1,70-3,97) e considerar ideal amamentar por menos de seis meses (RR: 1,67-3,27) representaram maior risco de desmame. A duração mediana do aleitamento materno foi cinco meses em 1980 e 11 meses em 2004.

Aleitamento Materno; Desmame; Promoção da Saúde; Fatores de Risco

\section{Referências}

1. Organização Mundial da Saúde. Estratégia global de "saúde para todos no ano 2000". Genebra: Organização Mundial da Saúde; 1981.

2. Rea MF. Reflexões sobre a amamentação no Brasil: de como passamos a 10 meses de duração. Cad Saúde Pública 2003; 19 Suppl 1:S37-45.

3. Cunningham AS, Jelliffe DB, Jelliffe EF. Breast-feeding and health in the 1980s: a global epidemiologic review. J Pediatr 1991; 118:659-66.

4. Lauer JA, Betrán AP, Victora CG, Onís M, Barros AJD. Breastfeeding patterns and exposure to suboptimal breastfeeding among children in developing countries: review and analysis of nationally representative surveys. BMC Med 2004; 2:26.

5. Oliveira MIC, Camacho LAB, Tedstone AE. Extending breastfeeding duration through primary care: a systematic review of prenatal and postnatal interventions. J Hum Lact 2001; 17:326-43.

\section{Colaboradores}

Os autores participaram conjuntamente de todas as etapas do estudo e da preparação do manuscrito.

\section{Agradecimentos}

À Gerente do Centro de Saúde São Marcos, Carmem Cadete pelo apoio institucional. Aos agentes comunitários de saúde do Centro de Saúde São Marcos e aos acadêmicos de medicina da Universidade Federal de Minas Gerais (UFMG), Juliana Almeida, Ana Ramos, Daniel Monteiro e Gustavo Maciel, pela contribuição na coleta e montagem do banco de dados e organização das tabelas. À Professora Conceição Cortês, do Departamento de Medicina Preventiva e Social da UFMG, pelas reflexões acerca do desenho metodológico deste estudo.
6. Alves CRL, Alvim CG, Magalhães MEN, Almeida JSCB, Goulart LMHF, Dias LS, et al. Saúde da criança e do adolescente: políticas públicas. In: Alves CRL, Viana MRA, organizadores. Saúde da família: cuidando de crianças e adolescentes. Belo Horizonte: Editora Coopmed; 2006. p. 7-14.

7. World Health Organization. Evidence for the ten steps to successful breastfeeding. Geneva: World Health Organization; 1998.

8. Fundo das Nações Unidas para a Infância. Iniciativa Hospital Amigo da Criança. http://www.unicef. org/brazil/ihac.htm (acessado em 21/Fev/2005).

9. Área de Saúde da Criança, Secretaria de Políticas de Saúde, Ministério da Saúde. Prevalência de aleitamento materno nas capitais brasileiras e no Distrito Federal. Brasília; Ministério da Saúde; 2001. 
10. Goulart EMA. Metodologia e informática na pesquisa médica. Belo Horizonte: Editora Coopmed; 2000.

11. Silveira FJF, Lamounier JA. Fatores associados à duração do aleitamento materno em três municípios do Alto Jequitinhonha, Minas Gerais, Brasil. Cad Saúde Pública 2006; 22:69-77.

12. Vobecky JS, Vobecky J, Froda S. The reliability of the maternal memory in a retrospective assessment of nutritional status. J Clin Epidemiol 1988; 41:261-5.

13. Goulart EMA. Análise da eficácia de um programa de incentivo ao aleitamento materno em um bairro periférico de Belo Horizonte - 1980/1986 [Dissertação de Mestrado]. Belo Horizonte: Faculdade de Medicina, Universidade Federal de Minas Gerais; 1988.

14. Giugliani ERJ. O aleitamento materno na prática clínica. J Pediatr 2000; 76:238-52.

15. Campos-Filho N, Franco ELF. Microcomputer-assisted univariate survival data analysis using Kaplan-Meier life table estimators. Comput Methods Programs Biomed 1988; 27:223-8.

16. Greenland S. Modeling and variable selection in epidemiologic analysis. Am J Public Health 1989; 79:340-9.

17. Campos-Filho N, Franco ELF. Microcomputerassisted multivariate survival data analysis using Cox's proportional hazards regression model. Comput Methods Programs Biomed 1990; 31:81-7.

18. Barros FC, Victora CG. Epidemiologia da saúde infantil - um manual para diagnósticos comunitários. São Paulo: Editora Hucitec/Fundo das Nações Unidas para a Infância; 1994.

19. Faleiros FTV, Trezza EMC, Carandina L. Aleitamento materno: fatores de influência na sua decisão e duração. Rev Nutr 2006; 19:623-30.

20. Berra S, Salbulsky J, Rajmil L, Passamonte R, Pronsato J, Butinof M. Correlates of breastfeeding duration in an urban cohort from Argentina. Acta Paediatr 2003; 92:952-7.

21. Bueno MB, Souza JMP, Souza SB, Paz SMRS, Gimeno SGA, Siqueira AAF. Riscos associados ao processo de desmame entre crianças nascidas em hospital universitário de São Paulo, entre 1998 e 1999: estudo de coorte prospectivo do primeiro ano de vida. Cad Saúde Pública 2003; 19:1453-60.
22. Campos CR. A produção da cidadania: construindo o SUS em Belo Horizonte. In: Campos CR, Malta DM, Reis AT, Santos AF, Merhy EE, organizadores. Sistema único de saúde em Belo Horizonte: reescrevendo o público. São Paulo: Editora Xamã; 1998. p. 11-30.

23. Vannuchi MTO, Thomson Z, Escuder MML, Tacla TGM, Vezozzo KMK, Castro LMCP, et al. Perfil do aleitamento materno em menores de um ano no município de Londrina, Paraná. Rev Bras Saúde Matern Infant 2005; 5:155-62.

24. Caldeira AP, Goulart EMA. Situação do aleitamento materno em Montes Claros (MG): estudo de uma amostra representativa. J Pediatr 2000; 76:65-72.

25. Taveras EM, Capra AM, Braverman PA, Jensvold NG, Escobar GJ, Lieu TA. Clinician support and psychosocial risk factors associated with breastfeeding discontinuation. Pediatrics 2003; 112:108-15.

26. Susin LR, Giugliani ER, Kummer SC, Maciel M, Simon C, Silveira LC. Does parental breastfeeding knowledge increase breastfeeding rates? Birth 1999; 26:149-56

27. Donath SM, Amir LH; ALSPAC Study Team. Relationship between prenatal infant feeding intention an initiation and duration of breastfeeding: a cohort study. Acta Paediatr 2003; 92:352-6.

28. Heinig MJ, Farley K. Development of effective strategies to support breastfeeding. J Hum Lact 2001; 17:293-4.

29. Bueno LGS, Teruya KM. Aconselhamento em amamentação e sua prática. J Pediatr (Rio J) 2004; 80 (5 Suppl):S126-30.

30. Venâncio SI, Monteiro CA. A tendência da prática de amamentação no Brasil nas décadas de 70 e 80 . Rev Bras Epidemiol 1998; 1:40-9.

31. Del Ciampo LA, Junqueira MJG, Ricco RG, Daneluzzi JC, Ferraz IS, Júnior CEM. Tendência secular do aleitamento materno em uma unidade de atenção primária à saúde materno-infantil em Ribeirão Preto, São Paulo. Rev Bras Saúde Matern Infant 2006; 6:391-6.

32. Silva SM, Brunken GS, França GVA, Escuder MM, Venancio SI. Evolução do aleitamento materno em uma capital da Região Centro-Oeste do Brasil entre 1999 e 2004. Cad Saúde Pública 2007; 23:1539-46.

Recebido em 11/Abr/2007

Versão final reapresentada em 30/Ago/2007

Aprovado em 19/Out/2007 